\title{
Blocking of Glucagonlike Peptide-1 Receptors in the Exocrine Pancreas Improves Specificity for $\beta$-Cells in a Mouse Model of Type 1 Diabetes
}

\author{
Eshita Khera*1, Liang Zhang*1, Sheryl Roberts ${ }^{2}$, Ian Nessler ${ }^{1}$, Darleen Sandoval ${ }^{3}$, Thomas Reiner ${ }^{2,4,5}$, and \\ Greg M. Thurber ${ }^{1,6}$ \\ ${ }^{1}$ Department of Chemical Engineering, University of Michigan, Ann Arbor, Michigan; ${ }^{2}$ Department of Radiology, Memorial Sloan \\ Kettering Cancer Center, New York, New York; ${ }^{3}$ Department of Surgery, University of Michigan Medical School, Ann Arbor, \\ Michigan; ${ }^{4}$ Department of Radiology, Weill Cornell Medical College, New York, New York; ${ }^{5}$ Chemical Biology Program, Memorial \\ Sloan Kettering Cancer Center, New York, New York; and ${ }^{6}$ Department of Biomedical Engineering, University of Michigan, Ann \\ Arbor, Michigan
}

\begin{abstract}
The diabetes community has long desired an imaging agent to quantify the number of insulin-secreting $\beta$-cells, beyond just functional equivalents (insulin secretion), to help diagnose and monitor early stages of both type 1 and type 2 diabetes mellitus. Loss in the number of $\beta$-cells can be masked by a compensatory increase in function of the remaining cells. Since $\beta$-cells form only about $1 \%$ of the pancreas and decrease as the disease progresses, only a few imaging agents, such as exendin, have demonstrated clinical potential to detect a drop in the already scarce signal. However, clinical translation of imaging with exendin has been hampered by pancreatic uptake that is higher than expected in subjects with long-term diabetes who lack $\beta$-cells. Exendin binds glucagonlike peptide-1 receptor (GLP-1R), previously thought to be expressed only on $\beta$-cells, but recent studies report low levels of GLP-1R on exocrine cells, complicating $\beta$-cell mass quantification. Methods: Here, we used a GLP-1R knockout mouse model to demonstrate that exocrine binding of exendin is exclusively via GLP-1R $(\sim 1,000 /$ cell) and not any other receptor. We then used lipophilic $\mathrm{Cy}-7$ exendin to selectively preblock exocrine GLP-1R in healthy and streptozotocininduced diabetic mice. Results: Sufficient receptors remain on $\beta$ cells for subsequent labeling with a fluorescent- or ${ }^{111} \mathrm{In}$-exendin. Conclusion: Selective GLP-1R blocking, which improves contrast between healthy and diabetic pancreata and provides a potential avenue for achieving the long-standing goal of imaging $\beta$-cell mass in the clinic.
\end{abstract}

Key Words: exendin; beta cell mass; exocrine GLP-1R; type 1 diabetes; streptozotocin; mouse model

J Nucl Med 2019; 60:1635-1641

DOI: 10.2967/jnumed.118.224881

$\mathbf{T}$

ype 1 diabetes mellitus, hereafter referred to as diabetes, is an autoimmune condition characterized by insulin-deficient hyperglycemia from immune-mediated destruction of $\beta$-cells. Given the

Received Dec. 13, 2018; revision accepted May 3, 2019.

For correspondence or reprints contact: Greg M. Thurber, University of Michigan, 2800 Plymouth Rd., Ann Arbor, MI 48109.

E-mail: gthurber@umich.edu

${ }^{*}$ Contributed equally to this work.

Published online May 10, 2019.

COPYRIGHT (C 2019 by the Society of Nuclear Medicine and Molecular Imaging. significant delay between $\beta$-cell loss and onset of hyperglycemia (1) and the excess capacity of insulin-secreting cells, isolating the impact of $\beta$-cell function from total $\beta$-cell mass (BCM) in the clinic is challenging. Current approaches to $\mathrm{BCM}$ detection are indirect, (e.g., measuring C-peptide) and cannot distinguish between a large $\mathrm{BCM}$ with low secretion and a small BCM with high secretion (i.e., a honeymoon phase (2)). Consequently, there is a critical need for direct and noninvasive mapping of BCM changes for early disease screening and progression monitoring. Molecular imaging of $\beta$-cells is a promising approach for measuring BCM but presents several unique challenges. $\beta$-cells constitute only a small fraction of the pancreas (1\%-2\% cells) and are organized in islets of Langerhans approximately $30-400 \mu \mathrm{m}$ in diameter (3), which is below the resolution of clinical scanners. Combined with partial-volume effects, the detectable signal from BCM is attenuated nearly 100-fold (3), even in healthy subjects.

Despite these hurdles, several probes directed toward $\beta$-cell markers, broadly classified as either functional or BCM probes, have recently progressed to studies in larger animals and clinical studies $(4,5)$. Functional probes cannot distinguish healthy BCM from a residual fraction of functionally overcompensating $\beta$-cells in the early stages of diabetes, for which treatment intervention may be most effective (1). Quantitative BCM probes can measure total $\mathrm{BCM}$ rather than only functionally active $\beta$-cells, and exendin probes directed against glucagonlike peptide-1 receptor (GLP-1R) (6) have emerged as promising candidates, possessing ideal imaging agent properties (7). Yet, advanced preclinical and clinical success for imaging has been surprisingly limited by off-target pancreatic uptake that is higher than expected in subjects with long-term diabetes.

GLP-1R on non- $\beta$-cells could explain the lack of clinical distinction between healthy volunteers and subjects with long-term diabetes using exendin-based probes (8). Although initially controversial because of cross-reactive antibodies combined with low absolute expression, several groups have verified measurable exocrine GLP-1R expression (8-20). Species-dependent variability in exocrine-to-islet signal ratio (Table 1) $(17,21)$ highlights additional challenges with evaluating exendin-based probes in animal models. We previously found an approximately 50-fold lower (accessible) GLP-1R expression on mouse exocrine cells than on $\beta$-cells (16), mediating significant non- $\beta$-cell uptake. Since poor clinical contrast is a key barrier to successful BCM imaging, we sought to address this issue using a quantitative approach. In this 
TABLE 1

Expression of GLP-1R on Exocrine Pancreas in Various Species

\begin{tabular}{lll}
\hline \multicolumn{1}{c}{ Species } & $\begin{array}{l}\text { Significant exocrine } \\
\text { GLP-1R expression }\end{array}$ & References \\
\hline Mouse & Yes & $14-16,21$ \\
Rat & No & $8,9,17,20,21$ \\
Pig & Yes & 18,21 \\
Nonhuman primate & Yes & 13,21 \\
Human & Yes & $9-13,19$ \\
\hline
\end{tabular}

study, using a GLP-1R knockout mouse model, we demonstrate that exocrine uptake of exendin is exclusively via GLP-1R. Furthermore, using a diabetes mouse model, we demonstrate as a proof of concept that selective blocking of exocrine GLP-1R using a slow-clearing exendin variant enables ${ }^{111} \mathrm{In}$-exendin to bind exclusively to $\beta$-cells, thereby providing higher specificity and accuracy for BCM quantification. With optimization and clinical measurements of GLP-1R expression, this strategy could potentially be translated to the clinic for quantifying subtle changes in BCM for presymptomatic diabetes detection and pretreatment disease monitoring.

\section{MATERIALS AND METHODS}

\section{Materials}

All chemicals were purchased from Sigma Aldrich, unless otherwise specified. Peptide and reaction details are provided in Supplemental Figure 1 (supplemental materials are available at http://jnm.snmjournals.org) $(16,22)$. All animal experiments were conducted under the approval of the Institutional Animal Care and Use Committee at the University of Michigan and Memorial Sloan Kettering Cancer Center and followed the National Institutes of Health guidelines for animal welfare. Healthy/wildtype (WT) C57BL/6J and streptozotocin-induced diabetic C57BL/6J mice (6-8 wk old) were purchased from Jackson Laboratory. Previously validated (23) GLP-1R knockout C57BL/6J mice (6-8 wk old) were used. Exendin conjugates were dosed via tail vein catheters.

\section{GLP-1R Knockout Experiments}

To probe the specificity of interaction between GLP-1R and exendin, healthy (WT) and GLP-1R knockout C57BL/6J mice were administered $1 \mathrm{nmol}$ of 647 -exendin either alone or preceded by a 15 -fold excess preblock dose of unlabeled exendin for $10 \min (n=3$ for each of the 4 conditions). After $20 \mathrm{~min}$, the mice were euthanized, and the pancreas was resected. Each pancreas was imaged macroscopically using a Licor Odyssey CLx imager to confirm successful islet targeting or blocking. Pancreata were then digested in a $1,000 \mathrm{U} / \mathrm{mL}$ concentration of collagenase IV for $15 \mathrm{~min}$ at $37^{\circ} \mathrm{C}$ with intermittent shaking. The digest solution was passed through a $40-\mu \mathrm{m}$ filter to generate a single-cell suspension and washed twice with cell medium and phosphate-buffered saline. Lastly, the cells were fixed in $4 \%$ paraformaldehyde, permeabilized, and stained for insulin using a rabbit anti-insulin primary and a goat anti-rabbit fluorescein isothiocyanate secondary antibody. Data were quantified using the Attune Acoustic Focusing Flow Cytometer (ThermoFisher) and analyzed using FlowJo (Becton, Dickinson and Co.). Events were gated for whole cells, followed by single cells, and finally for insulin-positive or -negative cells to distinguish distinct exocrine and $\beta$-cell populations. Statistical analysis using the Student $t$ test was performed on GraphPad Prism.

\section{Selective Blocking of Exocrine GLP-1R}

Each experiment consisted of a set of 3 mice administered a label dose, low-block dose, or high-block dose of the exendin conjugates $(n \geq 3)$ in healthy and streptozotocin-induced diabetic C57BL/6J mice (Table 2). The dosing schedule was optimized using modeling and experimental studies, accounting for previously observed exendin and receptor kinetics $(7,16)$. For the low-block group, a final 15-nmol WT-exendin dose (dose 3) was administered to quench any newly synthesized or recycled GLP-1R and prevent unwanted uptake during probe washout from the blood. At each endpoint, the mice were euthanized and the pancreas resected. For mice administered 647-exendin, the pancreas was processed in a similar manner as the GLP-1R knockout mice pancreas.

For mice administered ${ }^{111}$ In-exendin (Supplemental Table 1), the pancreata were resected and immediately exposed to a digital phosphor autoradiography plate overnight using a GE Healthcare Typhoon FLA 7000. Serial sections were used for immunohistochemistry to confirm tissue identity and insulin levels. Ex vivo biodistribution of ${ }^{111}$ In-exendin in healthy and streptozotocin-induced diabetic mice was performed. Briefly, blood and relevant organs were resected and weighed, and the radioactivity from each organ was counted in a Wizard automatic $\gamma$-counter (PerkinElmer). The organ accumulation was expressed as percentage injected dose per gram.

\section{Histology and Microscopy}

Low-block pancreata were embedded in optimal-cutting-temperature compound and flash-frozen in 2-methylbutane chilled using dry ice. Five-micrometer pancreas sections were fixed with $4 \%$ paraformaldehyde for $10 \mathrm{~min}$ at room temperature and stained overnight at

TABLE 2

Dosing and Intervals for Selective Exocrine GLP-1R Blocking

\begin{tabular}{|c|c|c|c|c|c|c|c|}
\hline Exendin type & Dose 1 & Interval (min) & Dose 2 & Interval (min) & Dose 3 & Interval (min) & \\
\hline \multicolumn{8}{|l|}{ Fluorescent } \\
\hline Label & $1 \mathrm{nmol}$ 647-exendin & 15 & Euthanize & & & & \\
\hline High block & $15 \mathrm{nmol}$ WT-exendin & 15 & $1 \mathrm{nmol} 647$-exendin & 15 & Euthanize & & \\
\hline Low block & $\begin{array}{l}7.39 \mathrm{pmol} / \mathrm{g} \text { Cy7- } \\
\text { exendin }\end{array}$ & 10 & $1 \mathrm{nmol}$ 647-exendin & 5 & $15 \mathrm{nmol}$ WT-exendin & 10 & Euthanize \\
\hline \multicolumn{8}{|l|}{ Radiolabeled } \\
\hline Label & $2.1 \mathrm{ng}{ }^{111} \mathrm{In}$-exendin & 60 & Euthanize & & & & \\
\hline High block & $15 \mathrm{nmol}$ WT-exendin & 15 & $2.1 \mathrm{ng}{ }^{111} \mathrm{In}$-exendin & 60 & Euthanize & & \\
\hline Low block & $\begin{array}{l}7.39 \mathrm{pmol} / \mathrm{g} \text { Cy7- } \\
\text { exendin }\end{array}$ & 10 & $2.1 \mathrm{ng}{ }^{111} \mathrm{In}$-exendin & 5 & $15 \mathrm{nmol}$ WT-exendin & 55 & Euthanize \\
\hline
\end{tabular}




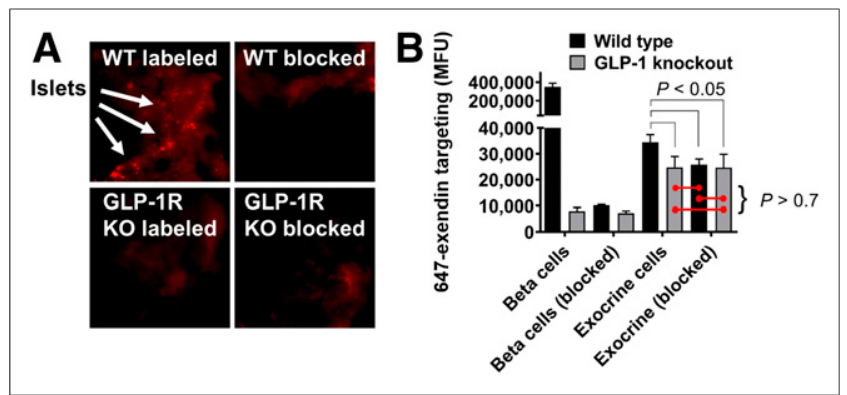

FIGURE 1. Knockout (KO) mouse model demonstrates exclusive exocrine binding of exendin to GLP-1R. (A) 647-exendin bound islets appear as punctate spots in WT labeled mice, which are absent in WT blocked, GLP-1R knockout labeled, and knockout blocked mice. (B) Quantitative flow cytometry analysis of exocrine pancreas cells shows statistically higher fluorescence in WT labeled mice than in WT blocked, knockout labeled, and knockout blocked mice, suggesting binding of exendin to exocrine cells exclusively via GLP-1R. MFU = median fluorescence unit.

$4^{\circ} \mathrm{C}$ with a rabbit anti-insulin primary antibody $(1: 150$ in phosphatebuffered saline with $0.1 \%$ bovine serum albumin). Sections were then incubated with goat antirabbit-AF555 secondary antibody (1:200 in phosphate-buffered saline with $0.1 \%$ bovine serum albumin) for $30 \mathrm{~min}$ at room temperature. Stained slices were imaged using an Olympus FV 1200 confocal microscope and analyzed on ImageJ.

\section{RESULTS}

\section{Exendin Uptake in Exocrine Pancreas Occurs Exclusively Via GLP-1R}

Macroscopic pancreas scans (Fig. 1A) demonstrated the presence of bright, punctate spot formation in a WT group administered 647-exendin only, consistent with strong targeting of $\beta$-cells in islets of Langerhans. Preblocked WT and both knockout groups did not form punctate spots, visually confirming the lack of interaction between GLP-1R and 647-exendin, either due to complete blocking or absence of GLP-1R. Quantitative single-cell analysis of exendin uptake by flow cytometry (Fig. 1B) demonstrated strong targeting of 647-exendin to endocrine $(\beta)$ cells in the labeled WT group, which diminished approximately 40-fold both with a preblock dose and when administered to knockout mice, consistent with the exclusive interaction between exendin and GLP-1R on $\beta$-cells. Comparatively, single-cell exocrine pancreas in the labeled WT group showed low yet robust 647-exendin targeting, which was also diminished with preblock exendin, indicating significant receptor-mediated binding on exocrine pancreas cells. Conversely, exocrine signal from both knockout groups was statistically nonsignificant from WT preblocked exocrine autofluorescence. The GLP-1R knockout model has fully functional islets with abrogated expression of only GLP-1R, whereas a nonfluorescent exendin dose blocks GLP-1R and any other cross-reacting receptors. Therefore, if exendin binding was not exclusive to GLP-1R, the exocrine signal from labeled GLP$1 \mathrm{R}$ knockout cells would have been higher than WT and GLP-1R preblocked cells. These results indicate that exendin binds exocrine pancreas exclusively via GLP-1R.

\section{Single-Cell Resolution of Selective Exocrine GLP-1R Blocking}

Direct administration of labeled exendin leads to binding of GLP-1R on both $\beta$-cells and exocrine cells. Although the differential in vivo expression between endocrine and exocrine cells is significant $(54,000$ GLP-1R per $\beta$-cell in C57B16/J mice (16) vs. 1,400 GLP-1R per exocrine cell), the higher prevalence of exocrine cells can dramatically skew BCM quantification $(16,21)$, since the limited spatial resolution of current imaging modalities cannot distinguish between $\beta$-cell signal and exocrine signal (Fig. $2 \mathrm{~A})$. Considering receptor expression levels, we hypothesized that a calibrated preblock dose of a slow-clearing Cy7-exendin could selectively occupy the scarce GLP-1R on exocrine cells while leaving sufficient GLP-1R on $\beta$-cells available for binding to the imaging probe and therefore accurately monitor the BCM loss (Fig. 2B).

Macroscopic scans of the 647-exendin group (Supplemental Fig. 2) showed bright punctate islets in healthy label pancreas that were absent in healthy high-block and streptozotocin-induced diabetic label, consistent with blocked or absent islets. Scans of healthy low-block pancreas still showed punctate islets, a promising indicator that preblocking with a low Cy7-exendin does not block all GLP-1R on $\beta$-cells. Flow cytometry analysis of the pancreata showed strong 647-exendin targeting in the small fraction of $\beta$-cells in healthy label pancreas, whereas healthy low-block pancreas showed a distinct (but decreased) $\beta$-cell signal compared with complete blocking in the healthy high-block pancreas (Fig. 3A). The tall exocrine peak for label is statistically higher $(P<$ $0.05)$ than either low block or high block, in both healthy and streptozotocin-induced
FIGURE 2. Simplified schematic for $\beta$-cell imaging. (A) $\beta$-cell quantification resolution using only imaging exendin probe (label). (B) Postulated improvement in $\beta$-cell quantification resolution using preblocked Cy7-exendin followed by imaging exendin probe (low block). 


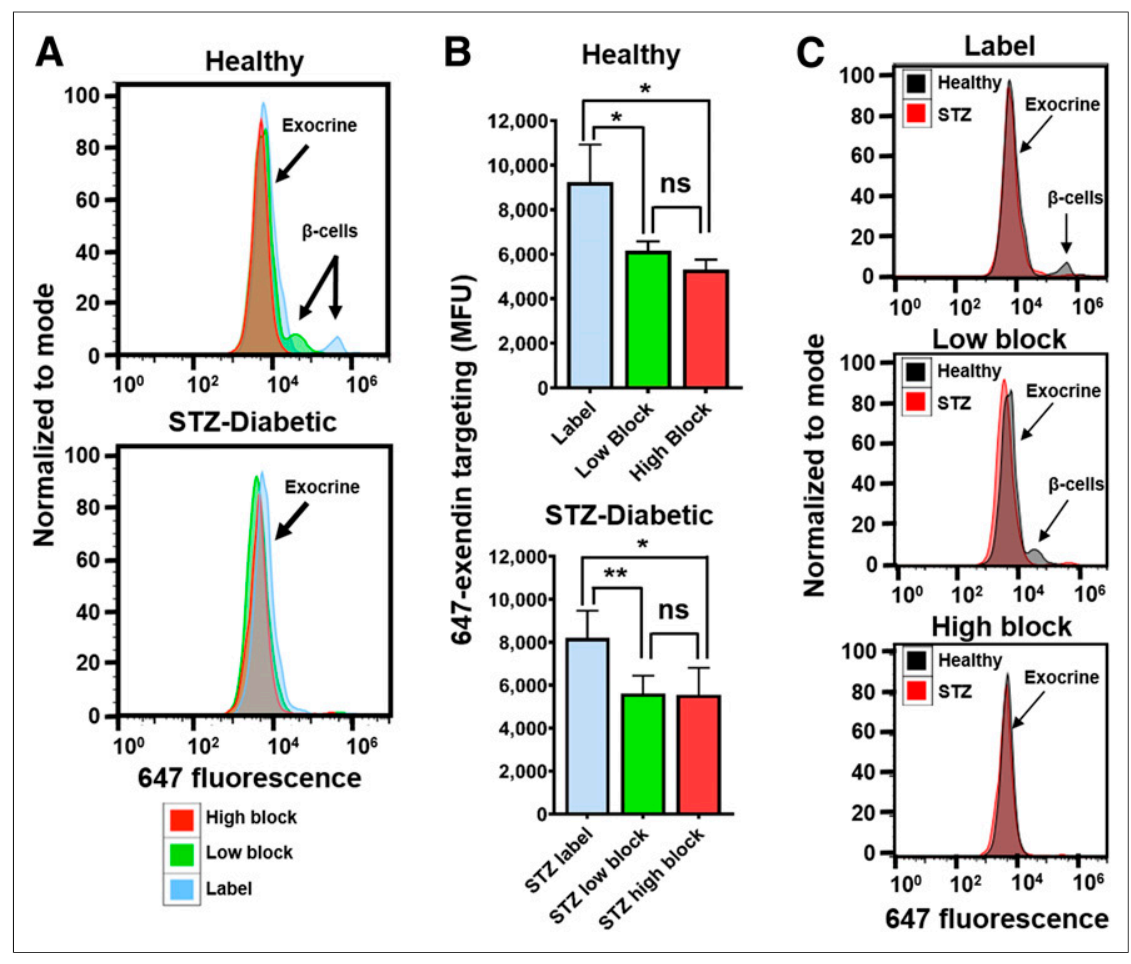

FIGURE 3. Selective exocrine GLP-1R blocking with single-cell resolution. (A) Fluorescent labeling of small fraction of $\beta$-cells observed in healthy label mice is retained in low-block mice but is completely absent in healthy high-block and all streptozotocin-induced diabetic mice. (B) Exocrine GLP-1R is completely blocked in both low-block and high-block pancreata in both healthy and streptozotocin-induced diabetic mice. (C) Healthy vs. streptozotocin-induced diabetic mice highlights improved resolution for detecting $\beta$-cell loss. ${ }^{\star} P<0.05$. ${ }^{\star \star} P<0.01$. MFU $=$ median fluorescence unit; ns $=$ not statistically significant; STZ = streptozotocin.

streptozotocin-induced diabetic pancreas was only 1.7-fold lower than label healthy pancreas, even though the former does not contain any $\beta$-cells. However, the contrast increased to 9.6-fold between low-block healthy and streptozotocin-induced diabetic pancreata, providing enhanced resolution of $\beta$-cell detection (Fig. 5A). This is in agreement with the sharp ( $\sim 12$-fold) reduction in ${ }^{111}$ In-exendin uptake in lowblock and high-block streptozotocin-induced diabetic pancreata $(P<0.005)$, highlighting the potential for low $\mathrm{Cy} 7$-exendin to block all exocrine GLP-1R. The healthy low-block pancreas exhibits ${ }^{111}$ In-exendin uptake intermediate to the label and high-block pancreata, consistent with complete blocking of exocrine GLP-1R but only partial $\beta$-cell GLP$1 \mathrm{R}$ blocking (Fig. 5B). For ${ }^{111}$ In-exendin in healthy and streptozotocin-induced diabetic high-block groups, uptake was slightly higher than expected (but not statistically significant) in healthy high block (Fig. 5B), as was likely due to rapid clearance of WT-exendin before ${ }^{111}$ In-exendin. Full organ biodistribution is included in Supplemental Fig. 4 (24,25).

For this proof-of-concept study, the Cy7exendin dose was optimized to err on the side of complete exocrine blocking, even at the cost of blocking more $\beta$-cell GLP-1R. Consequently, the difference in mean signal between healthy low block and streptozoto-

diabetic mice (Fig. 3B), demonstrating that direct labeling can significantly confound $\beta$-cell detection. By selectively blocking exocrine GLP-1R over $\beta$-cell GLP-1R, a low-block Cy7-exendin dose provides the best resolution for quantifying differences in BCM between healthy and diabetic pancreata (Fig. 3C).

\section{Improved Resolution of BCM Quantification Using ${ }^{111}$ In-Exendin Through Selective Blocking}

Since PET imaging of exendin in the pancreas is impractical in mice, ${ }^{111} \mathrm{In}$ was selected over PET probes such as ${ }^{68} \mathrm{Ga}$ for its longer half-life and ease of use for autoradiography, in addition to low background binding and high sensitivity. Whole-pancreas scans of ${ }^{111} \mathrm{In}$-exendin label streptozotocin-induced diabetic pancreata showed reduced radioactivity compared with healthy pancreata, consistent with the lack of $\beta$-cells, but still exhibited high diffuse signal, indicating nontargeted exocrine uptake (Fig. 4A). High-block pancreata exhibited low radioactivity in healthy and streptozotocin-induced diabetic mice (except when contaminated with fat tissue), reaffirming GLP-1R expression on exocrine cells. Low-block healthy pancreata exhibited reduced but measurable radioactivity, whereas the low-block streptozotocin-induced diabetic pancreata showed no radioactivity (similar to healthy $\mathrm{Cy} 7$ high block and streptozotocin-induced diabetic high block), highlighting the improved contrast between healthy and diabetic mice from selective blocking using Cy7-exendin. Histology confirmed the difference in number and size of insulin-positive islets between healthy and streptozotocin-induced diabetic mice (Fig. 4B; Supplemental Fig. 3). Quantitatively, ${ }^{111}$ In-exendin uptake in label cin-induced diabetic low block improved to 9.6-fold (Fig. 5A) but was not statistically significant. Most healthy low-block pancreata were higher than completely blocked signal-however, 2 of these were very high (2.4 and 2.6 percentage injected dose per gram). Though ideal, these 2 data points increased the SD for healthy low-block mice and drove the lack of statistical significance. If these 2 values are excluded, the resulting difference between lowblock healthy and streptozotocin-induced diabetic pancreata is still better than no blocking dose (3.6-fold with low block vs. 1.7-fold difference with label) and is statistically significant $(P<0.05)$ (Supplemental Fig. 5). Additional fine tuning of the Cy7-exendin dose in future studies may help alleviate this variability.

\section{DISCUSSION}

Quantifying BCM is an important goal in diabetes research, and exendin-based probes have many optimal properties. However, recent evidence shows low-level GLP-1R on exocrine cells, and this confounding effect from nontarget cells is a primary hurdle for clinical translation of exendin for BCM quantification. In this study, we confirmed that GLP-1R alone is responsible for the exocrine uptake. Additionally, our quantitative measurements show in a diabetes mouse model that selective exocrine GLP-1R blocking with a low cold-dose of lipophilic exendin can enhance the resolution of $\beta$-cells to distinguish healthy from diabetic pancreas.

Cold-dose blocking is routinely used in cancer imaging (26-28) for preferential blocking of receptors in healthy tissue to improve 

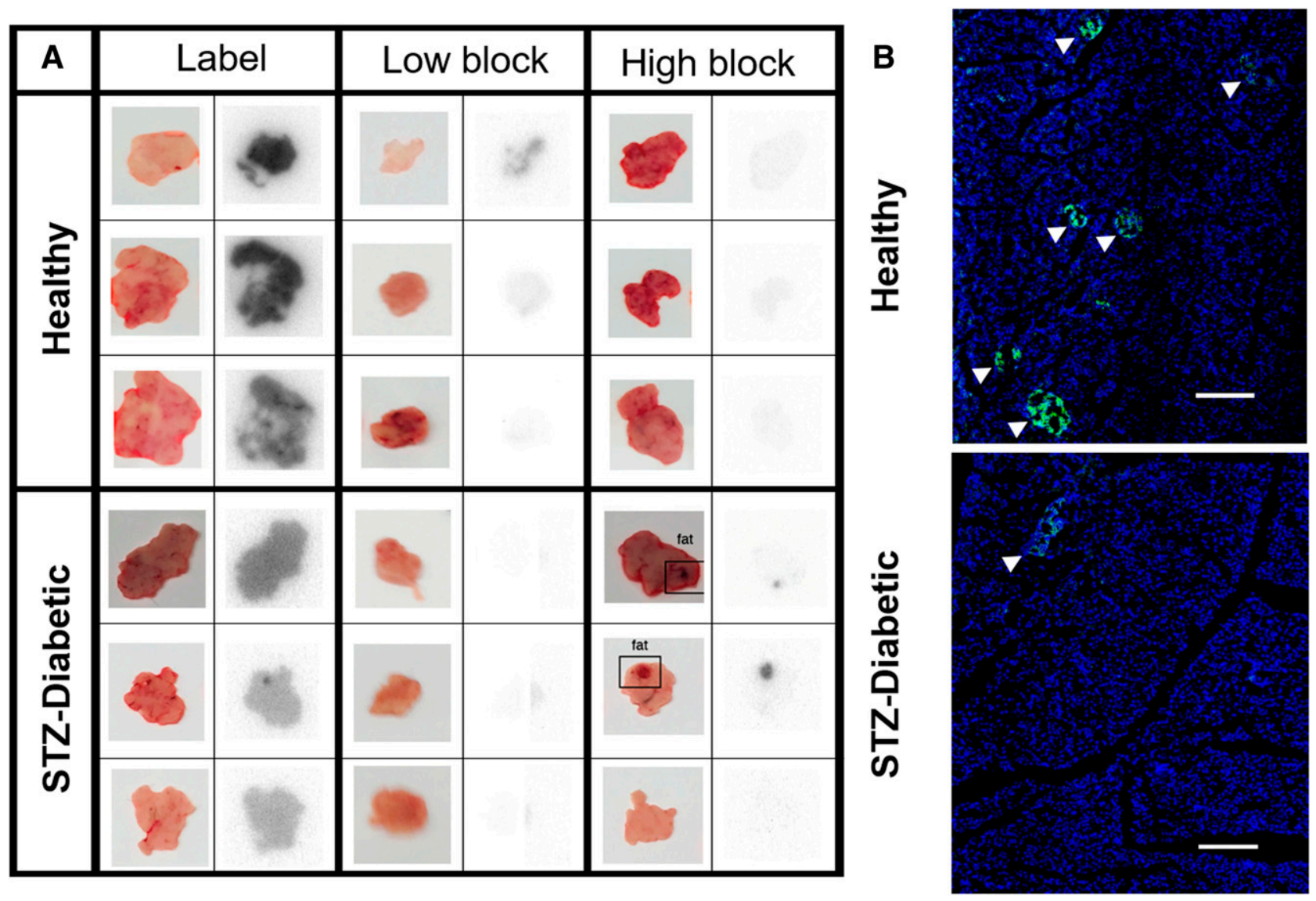

FIGURE 4. Selective blocking of exocrine GLP-1R isolates islet-specific radioactivity. (A) Macroscopic autoradiography of ${ }^{111}$ In-exendin injected mice shows that binding to exocrine GLP-1R can be abolished using Cy7-exendin low-block dose or high dose of WT-exendin. Tracer doses are generally not sufficient to see individual islets in thick tissue. (B) Insulin staining of pancreas slices confirms that healthy mice show numerous large islets (arrowheads) that are mostly absent in streptozotocin-induced diabetic mice. Scale bar $=250 \mu \mathrm{m}$.

contrast, facilitated by efficient normal-tissue uptake relative to poor tumor delivery. However, preferential blocking of exocrine over $\beta$-cells is more challenging, as they are in the same wellperfused pancreas. The blood vessel surface area-to-volume ratio (i.e., delivery) is 3-fold higher in the islets than in exocrine tissue $(3,29)$; thus, a subsaturating cold-block dose would theoretically block 3 -fold more $\beta$-cell receptors than exocrine receptors. Eriksson et al. found 4- and 5-fold higher exendin uptake in islets in mice and nonhuman primates, respectively (21), indicating that additional factors such as diffusion from the surrounding exocrine (similar to micrometastases (29)) could impact cold-dose blocking of exocrine GLP-1R. Additionally, receptor recycling is a critical consideration, and selective blocking of exocrine cells requires blocking both extracellular and intracellular reserves of exocrine GLP-1R with the preblock dose (Supplemental Fig. 6). To overcome these challenges, we used a lipophilic Cy7-exendin for blocking-first, to slow plasma clearance $(16,22)$ and block both extracellular and intracellular stores of exocrine GLP-1R (30) and, second, to slow exendin diffusion compared with hydrophilic exendin (Supplemental Fig. 7) (16,21,31), which limits its diffusion into islets from the surrounding exocrine tissue (Supplemental Fig. 8). Indeed, the use of unlabeled (hydrophilic) WT-exendin as the low preblock dose did not block exocrine GLP-1R as selectively as lipophilic Cy7-exendin (data not shown). Finally, the use of a lipophilic cyanine dye allowed tracking of the blocking probe if needed, whereas the similarity to the Food and Drug Administration-approved liraglutide (GLP-1 analog attached to a fatty acid instead of lipophilic $\mathrm{Cy} 7$ ) provides a potential path for translation. Though the doses used here are higher than therapeutic doses of liraglutide, several reports indicate that extremely high doses pose no safety issues or side effects besides nausea (resolved with antiemetics $(32,33)$ ). The systemic clearance of Cy7-exendin is faster than that of IgGs commonly used in cold-dose blocking, enabling repeat imaging if necessary. For clinical translation, the difference in expression between human exocrine- and $\beta$-cells needs to be sufficient to allow for selective blocking. This dosing window will compensate for variability in plasma clearance of the blocking dose by maintaining complete exocrine blocking while retaining sufficient $\beta$-cell GLP$1 \mathrm{R}$ to provide an excess of receptor for tracer probe binding.

In theory, a preblocking dose could reduce variability in human pancreas signal by eliminating the variable exocrine uptake, but differences in the pharmacokinetics of the preblocking dose have the potential to increase signal variability as well. Therefore, selection of an optimal blocking dose is critical. Too high a dose will block all GLP-1R, whereas too low a dose will leave exocrine GLP-1R unblocked. Here, we erred on the side of complete exocrine blocking at the cost of more endocrine blocking and variability. Further dose tuning could improve imaging resolution, but ultimately 


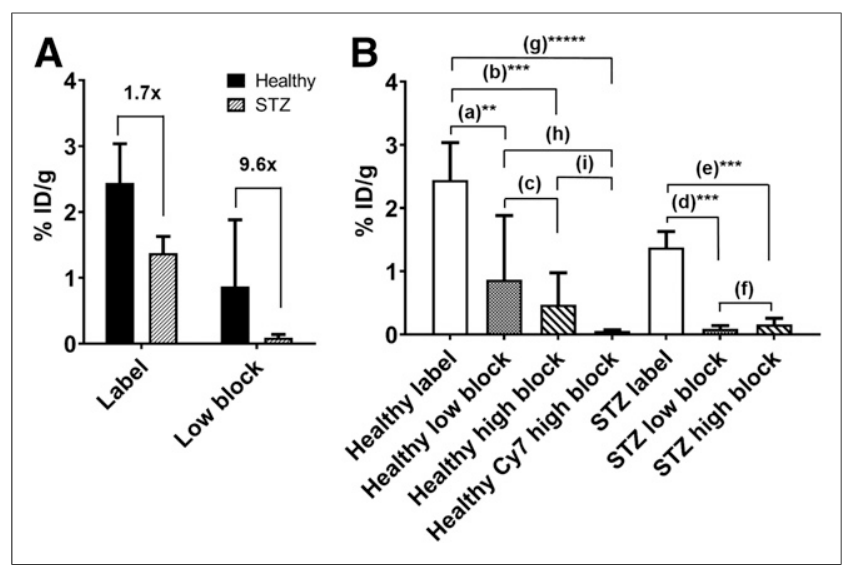

FIGURE 5. Quantification of BCM via selective ${ }^{111}$ In-exendin uptake. (A) Preblocking with lipophilic Cy7-exendin improves contrast between healthy and streptozotocin-induced diabetic pancreata ${ }^{111} \mathrm{In}$-exendin uptake from $1.7(P<0.05)$ to 9.6 (not statistically significant), improving resolution of $\beta$-cell imaging. (B) Uptake of ${ }^{111} \mathrm{In}$-exendin in streptozotocininduced diabetic mice is reduced with preblocking, but lipophilic Cy7exendin dose retains some uptake in healthy mice, indicating specific targeting of $\beta$-cells. ${ }^{\star \star} P<0.01$. ${ }^{\star \star \star} P<0.005$. ${ }^{\star \star \star \star \star} P<0.0005 . \% \mathrm{ID} / \mathrm{g}=$ percentage injected dose per gram. Fold differences: $a=2.8 ; b=5.2$; $c=1.8 ; d=15.3 ; e=8.5 ; f=0.56 ; g=43 ; h=15.3 ; i=8.3$.

clinical translation depends on relative islet-to-exocrine expression and delivery in humans and potential consideration of islet architecture (34). The differences in architecture, however, are unlikely to be significant given the extremely low penetration distance of lipophilic exendin $(10 \mu \mathrm{m})$ compared with hydrophilic exendin $(70 \mu \mathrm{m})$ (Supplemental Fig. 8). Promisingly, endocrine-to-exocrine expression appears similar between mice and humans (ex vivo isletto-exocrine ratio of $\sim 2-7$ in humans $(9,11,19), \sim 3$ in mice $(35)$, $\sim 5$ in monkeys (13), and $>30$ in rats (9)). However, histology does not capture delivery limitations and receptor trafficking present in vivo, requiring further assessment.

The use of radiolabeled PET/SPECT-based probes for BCM quantification is a contentious field, with concerns that relatively poor spatial resolution is a substantial limitation for accurate $\beta$-cell imaging. Since exocrine pancreas also expresses GLP-1R, PET/SPECT cannot distinguish whether the radioactivity is originating from $\beta$-cells or exocrine cells, thereby skewing the accuracy of BCM quantification $(36,37)$. However, as Gotthardt et al. pointed out, the key influencing factor for successful $\beta$-cell quantification using PET is not spatial resolution but ensuring that the radioactive signal observed is exclusively from $\beta$-cells (38). The use of lipophilic Cy7-exendin in this study achieves this precise BCM specificity by selectively blocking exocrine GLP-1R, thereby allowing ${ }^{111}$ In-exendin to bind only to $\beta$-cell GLP-1R. Thus, this dosing approach eliminates the confounding effects of exocrine uptake that have impeded the successful clinical translation of exendin imaging agents and achieves strong resolution of the BCM. Future developments of this method should focus on incorporation of PET imaging to evaluate clinical feasibility for $\beta$-cell imaging, which was not feasible in this proof-ofprinciple study because of the small size of the organs and the proximity of the kidneys relative to the diffusion distance of positrons in mice.

\section{CONCLUSION}

We present the novel approach of using exendin conjugates to improve the specificity of imaging $\beta$-cells to quantify BCM, despite the expression of GLP-1R on exocrine pancreas. Using a low preblock dose of slow-clearing lipophilic Cy7-exendin, we showed specific targeting of a 647 -exendin imaging probe to $\beta$-cells with single-cell resolution and a nearly 6 -fold improvement in $\beta$-cell versus exocrine uptake of ${ }^{111} \mathrm{In}$-exendin between healthy and diabetic mouse pancreata compared with administration of the imaging agent alone. With additional fine tuning and scaling of the selective blocking dose for expression, vascular density, and plasma clearance in humans, this imaging approach might serve as a foundation for clinical application that combines the sensitivity of PET with the strong $\beta$-cell resolution needed for accurate quantification of BCM.

\section{DISCLOSURE}

This work was supported by National Institutes of Health grants K01 DK093766 (Greg Thurber), R35 GM128819 (Greg Thurber), R01 CA204441 (Thomas Reiner), R43 CA228815 (Thomas Reiner), and P30 CA008748 and by an NSF graduate research fellowship (Ian Nessler). Thomas Reiner is a cofounder and shareholder of Summit Biomedical Imaging, LLC. No other potential conflict of interest relevant to this article was reported.

\section{ACKNOWLEDGMENTS}

We thank Dr. Dan Drucker for providing the GLP-1 receptor knockout mice and Dr. John Williams for assisting with breeding. We appreciate the support of Memorial Sloan Kettering Cancer Center's Animal Imaging Core Facility, Radiochemistry and Molecular Imaging Probes Core Facility. and Molecular Cytology Core Facility.

\section{KEY POINTS}

QUESTION: How do we overcome off-target uptake of diabetes imaging agents due to exocrine GLP-1R expression?

PERTINENT FINDINGS: We devised a novel dosing scheme that selectively preblocks exocrine GLP-1R over $\beta$-cell GLP-1R using a low dose of lipophilic Cy7-exendin. Proof-of-principle evaluation of this dosing scheme in a mouse model of type 1 diabetes confirmed selective exocrine blocking with single-cell resolution using a fluorescent exendin imaging agent and improved uptake contrast between healthy and diabetic mouse pancreata using 111 In-exendin.

IMPLICATIONS FOR PATIENT CARE: The study was designed with future translation to PET imaging in mind. Demonstration of enhanced contrast between healthy and diabetic mouse pancreata by selectively blocking exocrine GLP-1R paves the way for a potential clinical molecular imaging technique capable of BCM quantification for diabetes screening and progression monitoring.

\section{REFERENCES}

1. Atkinson MA, Eisenbarth GS. Type 1 diabetes: new perspectives on disease pathogenesis and treatment. Lancet. 2001;358:221-229.

2. Bowden SA. Partial remission (honeymoon phase) in type 1 diabetes mellitus. Frontiers Clin Drug Res Diabetes Obes. 2017;4:1-20.

3. Zhang L, Thurber GM. Imaging in diabetes. In: Lewis JS, Keshari KR, eds. Imaging and Metabolism. Cham, Switzerland: Springer International Publishing; 2018:175-197.

4. Wei W, Ehlerding EB, Lan X, Luo Q-y, Cai W. Molecular imaging of $\beta$-cells: diabetes and beyond. Adv Drug Deliv Rev. In press.

5. Jodal A, Schibli R, Béhé M. Targets and probes for non-invasive imaging of ß-cells. Eur J Nucl Med Mol Imaging. 2017;44:712-727. 
6. Manandhar B, Ahn J-M. Glucagon-like peptide-1 (GLP-1) analogs: recent advances, new possibilities, and therapeutic implications. J Med Chem. 2015; 58:1020-1037.

7. Zhang L, Bhatnagar S, Deschenes E, Thurber GM. Mechanistic and quantitative insight into cell surface targeted molecular imaging agent design. Sci Rep. 2016;6:25424.

8. Brom M, Woliner-van der Weg W, Joosten L, et al. Non-invasive quantification of the beta cell mass by SPECT with ${ }^{111}$ In-labelled exendin. Diabetologia. 2014; 57:950-959.

9. Waser B, Reubi JC. Radiolabelled GLP-1 receptor antagonist binds to GLP-1 receptor-expressing human tissues. Eur J Nucl Med Mol Imaging. 2014;41:11661171 .

10. Waser B, Blank A, Karamitopoulou E, Perren A, Reubi JC. Glucagon-like-peptide-1 receptor expression in normal and diseased human thyroid and pancreas. Mod Pathol. 2015;28:391-402.

11. Körner M, Stöckli M, Waser B, Reubi JC. GLP-1 receptor expression in human tumors and human normal tissues: potential for in vivo targeting. J Nucl Med. 2007;48:736-743.

12. Reubi JC, Perren A, Rehmann R, et al. Glucagon-like peptide-1 (GLP-1) receptors are not overexpressed in pancreatic islets from patients with severe hyperinsulinaemic hypoglycaemia following gastric bypass. Diabetologia. 2010;53:2641-2645.

13. Pyke C, Heller RS, Kirk RK, et al. GLP-1 receptor localization in monkey and human tissue: novel distribution revealed with extensively validated monoclonal antibody. Endocrinology. 2014;155:1280-1290.

14. Wewer Albrechtsen NJ, Albrechtsen R, Bremholm L, et al. Glucagon-like peptide 1 receptor signaling in acinar cells causes growth-dependent release of pancreatic enzymes. Cell Reports. 2016;17:2845-2856.

15. Hou Y, Ernst SA, Heidenreich K, Williams JA. Glucagon-like peptide-1 receptor is present in pancreatic acinar cells and regulates amylase secretion through cAMP. Am J Physiol Gastrointest Liver Physiol. 2016;310:G26-G33.

16. Zhang L, Thurber GM. Quantitative impact of plasma clearance and down-regulation on GLP-1 receptor molecular imaging. Mol Imaging Biol. 2016;18:79-89.

17. Willekens SMA, Joosten L, Boerman OC, et al. Strain differences determine the suitability of animal models for noninvasive in vivo beta cell mass determination with radiolabeled exendin. Mol Imaging Biol. 2016;18:705-714.

18. Nalin L, Selvaraju RK, Velikyan I, et al. Positron emission tomography imaging of the glucagon-like peptide-1 receptor in healthy and streptozotocin-induced diabetic pigs. Eur J Nucl Med Mol Imaging. 2014;41:1800-1810.

19. Kirk RK, Pyke C, von Herrath MG, et al. Immunohistochemical assessment of glucagon-like peptide 1 receptor (GLP-1R) expression in the pancreas of patients with type 2 diabetes. Diabetes Obes Metab. 2017;19:705-712.

20. Hörsch D, Goke R, Eissele R, Michel B, Goke B. Reciprocal cellular distribution of glucagon-like peptide-1 (GLP-1) immunoreactivity and GLP-1 receptor mRNA in pancreatic islets of rat. Pancreas. 1997;14:290-294.

21. Eriksson O, Rosenström U, Selvaraju RK, Eriksson B, Velikyan I. Species differences in pancreatic binding of DO3A-VS-Cys40-exendin4. Acta Diabetol. 2017;54:1039-1045.
22. Zhang L, Navaratna T, Thurber GM. A helix-stabilizing linker improves subcutaneous bioavailability of a helical peptide independent of linker lipophilicity. Bioconjug Chem. 2016;27:1663-1672.

23. Scrocchi LA, Brown TJ, MaClusky N, et al. Glucose intolerance but normal satiety in mice with a null mutation in the glucagon-like peptide 1 receptor gene. Nat Med. 1996;2:1254-1258.

24. Monazzam A, Lau J, Velikyan I, et al. Increased expression of GLP-1R in proliferating islets of men 1 mice is detectable by $\left[{ }^{68} \mathrm{Ga}\right] \mathrm{Ga}-\mathrm{DO} 3 \mathrm{~A}-\mathrm{VS}-\mathrm{Cys} 40$-exendin-4/PET. Sci Rep. 2018;8:748.

25. Viby N-E, Isidor MS, Buggeskov KB, Poulsen SS, Hansen JB, Kissow H. Glucagon-like peptide-1 (GLP-1) reduces mortality and improves lung function in a model of experimental obstructive lung disease in female mice. Endocrinology. 2013;154:4503-4511.

26. Houghton JL, Abdel-Atti D, Scholz WW, Lewis JS. Preloading with unlabeled CA19.9 targeted human monoclonal antibody leads to improved PET imaging with ${ }^{89} \mathrm{Zr}-5 \mathrm{~B} 1$. Mol Pharm. 2017;14:908-915.

27. Boswell CA, Mundo EE, Zhang C, et al. Differential effects of predosing on tumor and tissue uptake of an ${ }^{111}$ In-labeled anti-TENB2 antibody-drug conjugate. J Nucl Med. 2012;53:1454-1461.

28. Dijkers EC, Oude Munnink TH, Kosterink JG, et al. Biodistribution of ${ }^{89} \mathrm{Zr}$ trastuzumab and PET imaging of HER2-positive lesions in patients with metastatic breast cancer. Clin Pharmacol Ther. 2010;87:586-592.

29. Thurber GM, Weissleder R. Quantitating antibody uptake in vivo: conditional dependence on antigen expression levels. Mol Imaging Biol. 2011;13:623-632.

30. Eriksson O, Laughlin M, Brom M, et al. In vivo imaging of beta cells with radiotracers: state of the art, prospects and recommendations for development and use. Diabetologia. 2016;59:1340-1349.

31. Tung YC, Hsiao AY, Allen SG, Torisawa YS, Ho M, Takayama S. High-throughput 3D spheroid culture and drug testing using a 384 hanging drop array. Analyst. 2011;136:473-478.

32. Nakanishi R, Hirose T, Tamura Y, Fujitani Y, Watada H. Attempted suicide with liraglutide overdose did not induce hypoglycemia. Diabetes Res Clin Pract. 2013;99:e3-e4.

33. Bode SF, Egg M, Wallesch C, Hermanns-Clausen M. 10-fold liraglutide overdose over 7 months resulted only in minor side-effects. J Clin Pharmacol. 2013;53:785-786.

34. Kim A, Miller K, Jo J, Kilimnik G, Wojcik P, Hara M. Islet architecture: a comparative study. Islets. 2009;1:129-136.

35. Waser B, Reubi JC. Value of the radiolabelled GLP-1 receptor antagonist exendin(9-39) for targeting of GLP-1 receptor-expressing pancreatic tissues in mice and humans. Eur J Nucl Med Mol Imaging. 2011;38:1054-1058.

36. Alavi A, Werner TJ. Futility of attempts to detect and quantify beta cells by PET imaging in the pancreas: why it is time to abandon the approach. Diabetologia. 2018;61:2512-2515.

37. Cline GW, McCarthy TJ, Carson RE, Calle RA. Clinical and scientific value in the pursuit of quantification of beta cells in the pancreas by PET imaging. Diabetologia. 2018;61:2671-2673.

38. Gotthardt M, Eizirik DL, Aanstoot H-J, et al. Detection and quantification of beta cells by PET imaging: why clinical implementation has never been closer. Diabetologia. 2018;61:2516-2519. 